\title{
SCALING LIMIT OF THE CORRECTOR IN STOCHASTIC HOMOGENIZATION
}

\author{
JEAN-CHRISTOPHE MOURRAT AND JAMES NOLEN
}

\begin{abstract}
In the homogenization of divergence-form equations with random coefficients, a central role is played by the corrector. We focus on a discrete space setting and on dimension 3 and more. Completing the argument started in [11], we identify the scaling limit of the corrector, which is akin to a Gaussian free field.
\end{abstract}

MSC 2010: 35B27, 35J15, 35R60, 82D30.

KEYwORDS: stochastic homogenization, scaling limit, Gaussian free field.

\section{INTRODUCTION}

We consider a random conductance problem on $\mathbb{Z}^{d}$ associated with the discrete divergenceform operator $\nabla^{*} A \nabla$, where the coefficients of $A$ are independent, identically distributed random variables, bounded away from 0 and infinity. The main object of this paper is the stationary random corrector $\phi$, satisfying $\nabla^{*} A\left(\xi+\nabla \phi^{(\xi)}\right)=0$ with $\xi \in \mathbb{R}^{d}$ being a fixed vector, $d \geq 3$. This random function $\phi$ plays a central role $[8,2,5]$ in homogenenization theory for the operator $\nabla^{*} A \nabla$, a discrete analogue of the random elliptic operators considered in $[7,13]$. Our main result is that for $d \geq 3$ the appropriately rescaled corrector converges to a Gaussian field that has the homogeneity of a Gaussian free field.

To make this statement precise, we need to introduce some notation. We view $\mathbb{Z}^{d}$ as a graph with edges between nearest-neighbors, and we denote by $\mathbb{B}=\left\{(x, y) \in \mathbb{Z}^{d} \times \mathbb{Z}^{d}|| x-y \mid=1\right\}$ the set of (non-oriented) edges. Let $\left(\mathbf{e}_{1}, \ldots, \mathbf{e}_{d}\right)$ be the canonical basis of $\mathbb{Z}^{d}$. For every edge $e \in \mathbb{B}$, there exists a unique pair $(\underline{e}, i) \in \mathbb{Z}^{d} \times\{1, \ldots d\}$ such that $e$ links $\underline{e}$ to $\underline{e}+\mathbf{e}_{i}$. Given such a pair, we write $\bar{e}=\underline{e}+\mathbf{e}_{i}$. We call $\underline{e}$ the base point of the edge $e$. For $f: \mathbb{Z}^{\bar{d}} \rightarrow \bar{R}$, we let $\nabla f: \mathbb{B} \rightarrow \mathbb{R}$ be the gradient of $f$, defined by

$$
\nabla f(e)=f(\bar{e})-f(\underline{e}) .
$$

We write $\nabla^{*}$ for the formal adjoint of $\nabla$, that is, for $F: \mathbb{B} \rightarrow \mathbb{R}, \nabla^{*} F: \mathbb{Z}^{d} \rightarrow \mathbb{R}$ is defined via

$$
\left(\nabla^{*} F\right)(x)=\sum_{i=1}^{d} F\left(\left(x-\mathbf{e}_{i}, x\right)\right)-F\left(\left(x, x+\mathbf{e}_{i}\right)\right) .
$$

Given a family $\{a(e)\}_{e \in \mathbb{B}}$ of positive real numbers and a function $F: \mathbb{B} \rightarrow \mathbb{R}$, we let $A F(e)=$ $a(e) F(e)$. This provides us with a precise definition of the operator $\nabla^{*} A \nabla$.

In order to facilitate the derivation of the result (and match the assumptions made in [11] for the same reason), we make the simplifying assumption that the coefficients $\{a(e)\}_{e \in \mathbb{B}}$ are constructed as follows: we give ourselves a family $\left\{\zeta_{e}\right\}_{e \in \mathbb{B}}$ of independent standard Gaussian random variables, and define $a(e):=\mathbf{a}\left(\zeta_{e}\right)$, where $\mathbf{a}: \mathbb{R} \rightarrow \mathbb{R}$ is a twice differentiable function with bounded first and second derivatives, and taking values in a compact subset of $(0, \infty)$. We denote by $(\Omega, \mathbb{P})$ the underlying probability space and probability measure, and by $\langle\cdot\rangle$ the associated expectation.

Date: October 15, 2018. 
Gloria and Otto showed in [5] that for $d \geq 3$, for every given $\xi \in \mathbb{R}^{d}$, there exists a random, stationary $\phi^{(\xi)}: \mathbb{Z}^{d} \rightarrow \mathbb{R}$, called the corrector, satisfying

$$
\nabla^{*} A\left(\xi+\nabla \phi^{(\xi)}\right)=0 .
$$

Also, $\left\langle\left|\phi^{(\xi)}(0)\right|^{p}\right\rangle\left\langle\infty\right.$ for all $p \geq 1$. If we interpret the random variables $\{a(e)\}_{e \in \mathbb{B}}$ as conductances, the function $x \cdot \xi+\phi^{(\xi)}(x)$ represents a potential with macroscopic gradient $\xi$, and the value $a(e)\left(\xi+\nabla \phi^{(\xi)}\right)(e)$ represents a current across edge $e$. The effective conductivity of the network is the matrix $A_{\mathrm{h}}$ defined by

$$
\xi \cdot A_{\mathrm{h}} \xi=\sum_{e=\left(0, \mathbf{e}_{i}\right)}\left\langle\left(\xi+\nabla \phi^{(\xi)}\right)(e) a(e)\left(\xi+\nabla \phi^{(\xi)}\right)(e)\right\rangle .
$$

Let $\mathcal{G}_{\mathrm{h}}$ be the Green function of the continuum differential operator $-\nabla \cdot A_{\mathrm{h}} \nabla$. The main result of [11] is that there exists a $d \times d$ matrix $\mathrm{Q}^{(\xi)}$ and a constant $C<\infty$ such that letting

$$
\mathscr{K}_{\xi}(x):=\int_{\mathbb{R}^{d}} \nabla \mathcal{G}_{\mathrm{h}}(y) \cdot \mathrm{Q}^{(\xi)} \nabla \mathcal{G}_{\mathrm{h}}(x-y) \mathrm{d} y,
$$

we have

$$
\left|\left\langle\phi^{(\xi)}(0) \phi^{(\xi)}(x)\right\rangle-\mathscr{K}_{\xi}(x)\right| \leq C \frac{1 \vee \log ^{2}|x|}{|x|^{d-1}} \quad\left(x \in \mathbb{Z}^{d}\right) .
$$

The matrix $\mathbf{Q}^{(\xi)}$ is positive semi-definite, and $\mathbf{Q}_{j, k}^{(\xi)}$ is defined in terms of $\nabla \phi^{(\xi)}, \nabla \phi^{\left(\mathbf{e}_{j}\right)}$ and $\nabla \phi^{\left(\mathbf{e}_{k}\right)}$. We refer to [11] for a more precise description of the matrix $\mathrm{Q}^{(\xi)}$. The function $\mathscr{K}_{\xi}(x)$ has the same homogeneity as the Green function: $\mathscr{K}_{\xi}(\lambda x)=\lambda^{2-d} \mathscr{K}_{\xi}(x)$ for all $x \in \mathbb{R}^{d}, \lambda>0$.

In the present article, we show that $\phi^{(\xi)}$ converges to a Gaussian field. Following [10], for any $\alpha<0$, we denote by $\mathcal{C}_{\text {loc }}^{\alpha}=\mathcal{C}_{\text {loc }}^{\alpha}\left(\mathbb{R}^{d}\right)$ the (separable) local Hölder space with exponent of regularity $\alpha$. The following theorem is our main result:

Theorem 1.1 (Scaling limit of the corrector). Recall that we assume $d \geq 3$, and let $\Phi_{\varepsilon}^{(\xi)}$ be the (random) distribution defined by

$$
\Phi_{\varepsilon}^{(\xi)}(f):=\varepsilon^{\frac{d}{2}+1} \sum_{x \in \mathbb{Z}^{d}} f(\varepsilon x) \phi^{(\xi)}(x)
$$

for $f \in C_{c}^{\infty}$. For every $\alpha<1-d / 2$, the distribution $\Phi_{\varepsilon}^{(\xi)}$ converges in law to $\bar{\Phi}^{(\xi)}$ as $\varepsilon \rightarrow 0$ in the topology of $\mathcal{C}_{\text {loc }}^{\alpha}$, where $\bar{\Phi}^{(\xi)}$ is the Gaussian random field such that for every smooth, compactly supported function $f, \Phi^{(\xi)}(f)$ is a centred Gaussian with variance

$$
\sigma^{2}(f):=\int f(x) \mathscr{K}_{\xi}(y-x) f(y) \mathrm{d} x \mathrm{~d} y .
$$

In [1] (Conjecture 5), it was conjectured that the appropriate scaling limit for the corrector is a Gaussian free field. While Theorem 1.1 shows that the limit is a Gaussian field, the covariance structure can be different from the Gaussian free field, as explained in [11].

From (1.2), one infers that for every smooth and compactly supported function $f$, one has

$$
\operatorname{Var}\left[\Phi_{\varepsilon}^{(\xi)}(f)\right] \underset{\varepsilon \rightarrow 0}{\longrightarrow} \sigma^{2}(f) \text {. }
$$

In order to prove Theorem 1.1, it suffices to prove that $(1)$ the fluctuations of $\Phi_{\varepsilon}^{(\xi)}(f)$ are Gaussian, and that (2) the law of $\Phi_{\varepsilon}^{(\xi)}$ is tight in $\mathcal{C}_{\text {loc }}^{\alpha}$. For the first part, we will in fact be more precise and give a rate of convergence:

Proposition 1.2 (Gaussian fluctuations). Let $d_{K}$ denote the Kantorovich-Wasserstein distance

$$
d_{K}(X, W)=\sup \left\{\langle h(X)-h(W)\rangle \mid\left\|h^{\prime}\right\|_{\infty} \leq 1\right\},
$$


and let $Y$ be a standard Gaussian random variable. For every $f \in C_{c}^{\infty}$, we have

$$
d_{K}\left(\Phi_{\varepsilon}(f), \sigma(f) Y\right) \underset{\varepsilon \rightarrow 0}{\longrightarrow} 0 .
$$

Moreover, if $\sigma(f)>0$ and $\sigma_{\varepsilon}=\sqrt{\operatorname{Var}\left(\Phi_{\varepsilon}(f)\right)}$, then

$$
d_{K}\left(\sigma_{\varepsilon}^{-1} \Phi_{\varepsilon}(f), Y\right) \lesssim \varepsilon^{d / 2} \log |\varepsilon|
$$

as $\varepsilon \rightarrow 0$.

Proposition 1.2 is proved in Section 2. We then prove the following tightness result in Section 3.

Proposition 1.3 (Tightness). For every $\alpha<1-d / 2$, the sequence of (random) distributions $\left(\Phi_{\varepsilon}^{(\xi)}\right)_{\varepsilon \in(0,1]}$ is tight in $\mathcal{C}_{\text {loc }}^{\alpha}$.

Remark 1.4. Theorem 1.1 can be reformulated as the joint convergence in law of $\left(\Phi_{\varepsilon}^{\left(\mathbf{e}_{1}\right)}, \ldots, \Phi_{\varepsilon}^{\left(\mathbf{e}_{d}\right)}\right)$ to a Gaussian vector field. Indeed, tightness for the product topology follows from the tightness of each of the coordinates. The limit law is then uniquely identified since Theorem 1.1 gives a characterisation of the limit law of every linear combination of $\left(\Phi_{\varepsilon}^{\left(\mathbf{e}_{1}\right)}, \ldots, \Phi_{\varepsilon}^{\left(\mathbf{e}_{d}\right)}\right)$. The covariance structure of the limiting field can be inferred by a polarization (with respect to $\xi$ ) of the left-hand side of (1.2).

\section{Proof of Proposition 1.2}

From now on, we drop the dependence on $\xi$ in the notation for simplicity, writing $\phi$ and $\Phi_{\varepsilon}$ instead of $\phi^{(\xi)}$ and $\Phi_{\varepsilon}^{(\xi)}$, respectively.

If $\sigma(f)=0$, then the distribution of $\sigma(f) W$ is $\delta_{0}$. In this case, (1.5) follows immediately from Chebyshev's inequality and the fact that $\left\langle\Phi_{\varepsilon}(f)\right\rangle=0$ : for all Lipschitz functions $h$ with $\left\|h^{\prime}\right\| \leq 1$,

$$
\begin{aligned}
\left|\left\langle h\left(\Phi_{\varepsilon}(f)\right)\right\rangle-\langle h(\sigma(f) W)\rangle\right| & =\left|\left\langle h\left(\Phi_{\varepsilon}(f)\right)\right\rangle-h(0)\right| \\
& \leq\left\langle\left|h\left(\Phi_{\varepsilon}(f)\right)-h(0)\right|\right\rangle \\
& \leq\left\|h^{\prime}\right\|\left\langle\left|\Phi_{\varepsilon}(f)\right|\right\rangle \leq \sqrt{\operatorname{Var}\left(\Phi_{\varepsilon}(f)\right)}
\end{aligned}
$$

Hence,

$$
d_{K}\left(\Phi_{\varepsilon}(f), \sigma(f) W\right) \leq \sqrt{\operatorname{Var}\left(\Phi_{\varepsilon}(f)\right)} \rightarrow 0
$$

holds in this case.

So let us suppose that $\sigma(f)>0$. We wish to prove (1.5) and (1.6). Our proof will be based on the following proposition, which is a version of Theorem 2.2 in [4] and Theorem 3.1 (and Remark 3.6) of [12], stated in a form that is convenient for our purpose.

For a random variable $F \in L^{2}(\Omega)$ we say that $U=\partial_{e} F \in L^{2}(\Omega)$ is the weak derivative with respect to $\zeta_{e}$ (recall that $\zeta \sim N(0,1)$ ) if the following holds : for any finite subset $\Lambda \subset \mathbb{B}$ and any smooth, compactly supported function $\eta: \mathbb{R}^{|\Lambda|} \rightarrow \mathbb{R}$, we have

$$
\langle U \eta(\zeta)\rangle=\left\langle F \zeta_{e} \eta(\zeta)\right\rangle-\left\langle F \frac{\partial \eta}{\partial \zeta_{e}}(\zeta)\right\rangle
$$

where $\eta(\zeta)$ depends only on $\left\{\zeta_{e^{\prime}}\right\}_{e^{\prime} \in \Lambda}$.

Proposition 2.1. Let $F \in L^{2}(\Omega)$ be such that $\langle F\rangle=0,\left\langle F^{2}\right\rangle=1$. Assume also that $F$ has weak derivatives satisfying $\sum_{e}\left\langle\left|\partial_{e} F\right|^{4}\right\rangle^{1 / 2}<\infty$ and $\left\langle\left|\partial_{e^{\prime}} \partial_{e} F\right|^{4}\right\rangle<\infty$ for all e, $e^{\prime} \in \mathbb{B}$. Let $Y \sim N(0,1)$. Then

$$
\sup _{\left\|h^{\prime}\right\|_{\infty} \leq 1}\langle h(F)-h(Y)\rangle \leq \sqrt{\frac{5}{\pi}} \sqrt{\sum_{e^{\prime}}\left(\sum_{e}\left\langle\left|\partial_{e} F\right|^{4}\right\rangle^{1 / 4}\left\langle\left|\partial_{e^{\prime}} \partial_{e} F\right|^{4}\right\rangle^{1 / 4}\right)^{2}} .
$$


A proof of Proposition 2.1 is given later in Section 4. We note that except for the numerical constant, the same result holds if the weak derivatives are replaced by the so-called Glauber derivatives, hence providing a version of the result that applies to functions of independent random variables that are not necessarily Gaussian, see Remark 2.3 of [11].

We will apply this proposition to $F=\sigma_{\varepsilon}^{-1} \Phi_{\varepsilon}(f)$, where $\sigma_{\varepsilon}=\sqrt{\operatorname{Var}\left(\Phi_{\varepsilon}(f)\right)} \rightarrow \sigma(f)>0$. Without loss of generality, we may assume that $f(x)=0$ when $|x|>1$. For any edges $e, e^{\prime}$,

$$
\partial_{e} \Phi=\varepsilon^{\frac{d}{2}+1} \sum_{x \in \mathbb{Z}^{d}} f(\varepsilon x) \partial_{e} \phi(x),
$$

and

$$
\partial_{e^{\prime}} \partial_{e} \Phi=\varepsilon^{\frac{d}{2}+1} \sum_{x \in \mathbb{Z}^{d}} f(\varepsilon x) \partial_{e^{\prime}} \partial_{e} \phi(x) .
$$

We recall from [5] that the stationary function $\phi$ is defined by a limit

$$
\phi(0)=\lim _{\mu \rightarrow 0} \phi_{\mu}(0), \quad \text { in } L^{p}(\Omega)
$$

for every $p \geq 1$, where the stationary process $\phi_{\mu}(x)$ satisfies the regularized corrector problem

$$
-\mu \phi_{\mu}+\nabla^{*} A\left(\xi+\nabla \phi_{\mu}\right)=0
$$

with $\mu>0$. The random variable $\phi_{\mu}(0)$, with $\mu>0$, may be regarded as a function of the random variables $\left\{\zeta_{e}\right\}$ (as a Borel measureable function on $\mathbb{R}^{\mathbb{B}}$ ), and in [5] the weak derivatives $\partial_{e} \phi_{\mu}$ were shown to exist, coinciding with $\frac{\partial \phi_{\mu}}{\partial \zeta_{e}}$. Although the limit $\phi(0) \in L^{p}(\Omega)$ is not defined as a function of the $\left\{\zeta_{e}\right\}$ (it is only defined on a set of probability one), the weak derivatives $\partial_{e} \phi$ and $\partial_{e} \partial_{e^{\prime}} \phi$ may be shown to exist through the limit as $\mu \rightarrow 0$, in the usual way. (Refer to Remark 4.8 of [11] for more about this point.)

For $e=\left[z, z+\mathbf{e}_{\mathbf{i}}\right], \partial_{e} \phi$ satisfies

$$
-\nabla^{*} \cdot a \nabla\left(\partial_{e} \phi\right)=\nabla^{*} \cdot\left(\partial_{e} a\right)(\nabla \phi+\xi)
$$

and thus

$$
\partial_{e} \phi(x)=-\left(\partial_{e} a(e)\right) \nabla_{e} G(x, e)\left(\nabla \phi(e)+\xi_{e}\right),
$$

where $G$ denotes the Green function associated with the operator $\nabla^{*} A \nabla$. We use $\nabla_{e} G(x, e)$ to denote $G\left(x, z+\mathbf{e}_{\mathbf{i}}\right)-G(x, z)$ where $e=\left[z, z+\mathbf{e}_{\mathbf{i}}\right]$. Similarly, if $e^{\prime}=\left[y, y+\mathbf{e}_{\mathbf{j}}\right]$, then $\partial_{e^{\prime}} \partial_{e} \phi$ satisfies (2.4) $-\nabla^{*} \cdot a \nabla\left(\partial_{e^{\prime}} \partial_{e} \phi\right)=\nabla^{*} \cdot\left(\partial_{e^{\prime}} \partial_{e} a\right)(\nabla \phi+\xi)+\nabla^{*} \cdot\left(\partial_{e} a(e)\right)\left(\nabla \partial_{e^{\prime}} \phi\right)+\nabla^{*} \cdot\left(\partial_{e^{\prime}} a(e)\right)\left(\nabla \partial_{e} \phi\right)$.

For $e \neq e^{\prime}, \partial_{e^{\prime}} \partial_{e} a=0$, and we have

$$
\begin{aligned}
\partial_{e^{\prime}} \partial_{e} \phi(x)= & -\left(\partial_{e} a(e)\right) \nabla_{e} G(x, e)\left(\nabla_{e} \partial_{e^{\prime}} \phi(z)\right)-\left(\partial_{e^{\prime}} a\left(e^{\prime}\right)\right) \nabla_{e^{\prime}} G\left(x, e^{\prime}\right)\left(\nabla_{e^{\prime}} \partial_{e} \phi(y)\right) \\
= & \left(\partial_{e} a(e)\right)\left(\partial_{e^{\prime}} a\left(e^{\prime}\right)\right) \nabla_{e} G(x, e)\left(\nabla_{e} \nabla_{e^{\prime}} G\left(e, e^{\prime}\right)\left(\nabla \phi\left(e^{\prime}\right)+\xi_{e^{\prime}}\right)\right) \\
& +\left(\partial_{e} a(e)\right)\left(\partial_{e^{\prime}} a\left(e^{\prime}\right)\right) \nabla_{e^{\prime}} G\left(x, e^{\prime}\right)\left(\nabla_{e^{\prime}} \nabla_{e} G\left(e^{\prime}, e\right)\left(\nabla \phi(e)+\xi_{e}\right)\right),
\end{aligned}
$$

while for $e^{\prime}=e$, we have

$$
\begin{aligned}
\partial_{e}^{2} \phi(x)= & 2\left(\partial_{e} a(e)\right)^{2} \nabla_{e} G(x, e)\left(\nabla_{e} \nabla_{e} G(e, e)\left(\nabla_{e} \phi(e)+\xi_{e}\right)\right) \\
& -\left(\partial_{e}^{2} a(e)\right) \nabla_{e} G(x, e)\left(\nabla_{e} \phi(e)+\xi_{e}\right) .
\end{aligned}
$$

Therefore, applying the generalized Hölder inequality and the fact that $\left|\partial_{e} a\right|=\left|\mathbf{a}^{\prime}\left(\zeta_{e}\right)\right| \lesssim 1$ and $\left|\partial_{e}^{2} a\right|=\left|\mathbf{a}^{\prime \prime}\left(\zeta_{e}\right)\right| \lesssim 1$

$$
\begin{aligned}
\left\langle\left|\partial_{e} \partial_{e^{\prime}} \phi(x)\right|^{p}\right\rangle^{1 / p} \lesssim & \left\langle\left|\nabla_{e} G(x, e)\right|^{3 p}\right\rangle^{\frac{1}{3 p}}\left\langle\left|\nabla_{e} \nabla_{e^{\prime}} G\left(e, e^{\prime}\right)\right|^{3 p}\right\rangle^{\frac{1}{3 p}}\left\langle\left|\nabla \phi\left(e^{\prime}\right)+\xi\right|^{3 p}\right\rangle^{\frac{1}{3 p}} \\
& +\left\langle\left|\nabla_{e} G\left(x, e^{\prime}\right)\right|^{3 p}\right\rangle^{\frac{1}{3 p}}\left\langle\left|\nabla_{e^{\prime}} \nabla_{e} G\left(e^{\prime}, e\right)\right|^{3 p}\right\rangle^{\frac{1}{3 p}}\left\langle|\nabla \phi(e)+\xi|^{3 p}\right\rangle^{\frac{1}{3 p}} .
\end{aligned}
$$

Also,

$$
\left\langle\left|\partial_{e} \phi(x)\right|^{p}\right\rangle^{1 / p} \lesssim\left\langle\left|\nabla_{e} G(x, e)\right|^{2 p}\right\rangle^{\frac{1}{2 p}}\left\langle|\nabla \phi(x)+\xi|^{2 p}\right\rangle^{\frac{1}{2 p}}
$$


Gloria and Otto [5] proved that for all $p \geq 1$,

$$
\left\langle|\nabla \phi(e)+\xi|^{p}\right\rangle^{1 / p} \lesssim 1
$$

Marahrens and Otto [9] proved that the Green function satisfies

$$
\left\langle\left|\nabla_{e} G(0, e)\right|^{p}\right\rangle^{1 / p} \lesssim \frac{1}{(1+|e|)^{d-1}},
$$

and

$$
\left\langle\left|\nabla_{e^{\prime}} \nabla_{e} G\left(e^{\prime}, e\right)\right|^{p}\right\rangle^{1 / p} \lesssim \frac{1}{\left(1+\left|e-e^{\prime}\right|\right)^{d}}
$$

for all $p \geq 2$. By combining these crucial estimates with (2.7) and (2.10), we obtain

$$
\left\langle\left|\partial_{e} \partial_{e^{\prime}} \phi(x)\right|^{p}\right\rangle^{1 / p} \lesssim\left(\frac{1}{(1+|x-e|)^{d-1}}+\frac{1}{\left(1+\left|x-e^{\prime}\right|\right)^{d-1}}\right) \cdot \frac{1}{\left(1+\left|e-e^{\prime}\right|\right)^{d}},
$$

and

$$
\left\langle\left|\partial_{e} \phi(x)\right|^{p}\right\rangle^{1 / p} \lesssim \frac{1}{(1+|x-e|)^{d-1}}
$$

Applying (2.10) and (2.9) to $\partial_{e} \Phi$ and $\partial_{e^{\prime}} \partial_{e} \Phi$, and recalling that $f(x)=0$ when $|x|>1$, we have

$$
\begin{aligned}
\varepsilon^{-\frac{d}{2}-1}\left\langle\left|\partial_{e} \Phi\right|^{p}\right\rangle^{1 / p} & \left.\left.\lesssim \sum_{|x| \leq \varepsilon^{-1}}\langle| \partial_{e} \phi(x)\right)\left.\right|^{p}\right\rangle^{1 / p} \\
& \lesssim \sum_{|x| \leq \varepsilon^{-1}} \frac{1}{(1+|x-e|)^{d-1}},
\end{aligned}
$$

and

$$
\begin{aligned}
\varepsilon^{-\frac{d}{2}-1}\left\langle\left|\partial_{e} \partial_{e^{\prime}} \Phi\right|^{p}\right\rangle^{1 / p} & \left.\left.\lesssim \sum_{|x| \leq \varepsilon^{-1}}\langle| \partial_{e} \partial_{e^{\prime}} \phi(x)\right)\left.\right|^{p}\right\rangle^{1 / p} \\
& \lesssim \sum_{|x| \leq \varepsilon^{-1}}\left(\frac{1}{(1+|x-e|)^{d-1}}+\frac{1}{\left(1+\left|x-e^{\prime}\right|\right)^{d-1}}\right) \cdot \frac{1}{\left(1+\left|e-e^{\prime}\right|\right)^{d}}
\end{aligned}
$$

Now we are prepared to apply Proposition 2.1. The fact that $\sum_{e}\left\langle\left|\partial_{e} \Phi\right|^{4}\right\rangle^{1 / 2}<\infty$ and $\left\langle\left|\partial_{e^{\prime}} \partial_{e} \Phi\right|^{4}\right\rangle<\infty$ follows from (2.11), (2.12), and the following lemma:

Lemma 2.2. For all $e \in \mathbb{Z}^{d}$ and $\varepsilon \in(0,1]$.

$$
\sum_{|x| \leq \varepsilon^{-1}} \frac{1}{(1+|x-e|)^{d-1}} \lesssim \frac{\varepsilon^{-1}}{(1+|\varepsilon e|)^{d-1}}
$$

By the estimates above, we have

$$
\begin{aligned}
& \varepsilon^{-d-2} \sum_{e}\left\langle\left|\partial_{e} \partial_{e^{\prime}} \Phi\right|^{4}\right\rangle^{1 / 4}\left\langle\left|\partial_{e} \Phi\right|^{4}\right\rangle^{1 / 4} \\
& \quad \leq \sum_{e}\left(\sum_{|x| \leq \varepsilon^{-1}}\left(\frac{1}{(1+|x-e|)^{d-1}}+\frac{1}{\left(1+\left|x-e^{\prime}\right|\right)^{d-1}}\right) \cdot \frac{1}{\left(1+\left|e-e^{\prime}\right|\right)^{d}}\right)\left(\sum_{|x| \leq \varepsilon^{-1}} \frac{1}{(1+|x-e|)^{d-1}}\right) \\
& \quad=\sum_{e} \frac{1}{\left(1+\left|e-e^{\prime}\right|\right)^{d}}\left(\sum_{|x| \leq \varepsilon^{-1}}\left(\frac{1}{(1+|x-e|)^{d-1}}+\frac{1}{\left(1+\left|x-e^{\prime}\right|\right)^{d-1}}\right)\right)\left(\sum_{|x| \leq \varepsilon^{-1}} \frac{1}{(1+|x-e|)^{d-1}}\right) .
\end{aligned}
$$


Therefore,

$$
\begin{aligned}
& \varepsilon^{-d-2} \sum_{e}\left\langle\left|\partial_{e} \partial_{e^{\prime}} \Phi\right|^{4}\right\rangle^{1 / 4}\left\langle\left|\partial_{e} \Phi\right|^{4}\right\rangle^{1 / 4} \\
& \quad \leq \varepsilon^{-2} \sum_{e} \frac{1}{\left(1+\left|e-e^{\prime}\right|\right)^{d}}\left(\frac{1}{(1+|\varepsilon e|)^{d-1}}+\frac{1}{\left(1+\left|\varepsilon e^{\prime}\right|\right)^{d-1}}\right) \frac{1}{(1+|\varepsilon e|)^{d-1}} .
\end{aligned}
$$

Lemma 2.3. Let $p>0$. For all $e^{\prime} \in \mathbb{Z}^{d}$ and $\varepsilon \in(0,1 / 2]$,

$$
\sum_{e} \frac{1}{\left(1+\left|e-e^{\prime}\right|\right)^{d}} \frac{1}{(1+|\varepsilon e|)^{p}} \lesssim \frac{|\log \varepsilon|}{\left(1+\left|\varepsilon e^{\prime}\right|\right)^{d}}+\frac{|\log \varepsilon|}{\left(1+\left|\varepsilon e^{\prime}\right|\right)^{p}}
$$

Using Lemma 2.3, we conclude that

$$
\sum_{e} \frac{1}{\left(1+\left|e-e^{\prime}\right|\right)^{d}} \frac{1}{(1+|\varepsilon e|)^{2(d-1)}} \lesssim \frac{|\log \varepsilon|}{\left(1+\left|\varepsilon e^{\prime}\right|\right)^{d}}+\frac{|\log \varepsilon|}{\left(1+\left|\varepsilon e^{\prime}\right|\right)^{2(d-1)}} \lesssim \frac{|\log \varepsilon|}{\left(1+\left|\varepsilon e^{\prime}\right|\right)^{d}}
$$

and

$$
\sum_{e} \frac{1}{\left(1+\left|e-e^{\prime}\right|\right)^{d}} \frac{1}{(1+|\varepsilon e|)^{d-1}} \lesssim \frac{|\log \varepsilon|}{\left(1+\left|\varepsilon e^{\prime}\right|\right)^{d}}+\frac{|\log \varepsilon|}{\left(1+\left|\varepsilon e^{\prime}\right|\right)^{d-1}} \lesssim \frac{|\log \varepsilon|}{\left(1+\left|\varepsilon e^{\prime}\right|\right)^{d-1}}
$$

These bounds combined with (2.14) imply that

$$
\begin{aligned}
\varepsilon^{-2 d-4} \sum_{e^{\prime}}\left(\sum_{e}\left\langle\left|\partial_{e} \partial_{e^{\prime}} \Phi\right|^{4}\right\rangle^{1 / 4}\left\langle\left|\partial_{e} \Phi\right|^{4}\right\rangle^{1 / 4}\right)^{2} \lesssim & \varepsilon^{-4} \sum_{e^{\prime}}\left(\frac{|\log \varepsilon|}{\left(1+\left|\varepsilon e^{\prime}\right|\right)^{d}}\right)^{2} \\
& +\varepsilon^{-4} \sum_{e^{\prime}} \frac{1}{\left(1+\left|\varepsilon e^{\prime}\right|\right)^{2(d-1)}}\left(\frac{|\log \varepsilon|}{\left(1+\left|\varepsilon e^{\prime}\right|\right)^{d-1}}\right)^{2} \\
& \lesssim \varepsilon^{-4-d}(\log \varepsilon)^{2} .
\end{aligned}
$$

In view of this estimate, we see that the random variable $F_{\varepsilon}=\sigma_{\varepsilon}^{-1} \Phi_{\varepsilon}$ satisfies

$$
\sum_{e^{\prime}}\left(\sum_{e}\left\langle\left|\partial_{e} \partial_{e^{\prime}} F_{\varepsilon}\right|^{4}\right\rangle^{1 / 4}\left\langle\left|\partial_{e} F_{\varepsilon}\right|^{4}\right\rangle^{1 / 4}\right)^{2} \lesssim \sigma_{\varepsilon}^{-4} \varepsilon^{d}(\log \varepsilon)^{2}
$$

By Proposition 2.1, we conclude that

$$
\sup _{\left\|h^{\prime}\right\|_{\infty} \leq 1}\left\langle h\left(F_{\varepsilon}\right)-h(Y)\right\rangle \lesssim \sigma_{\varepsilon}^{-2} \varepsilon^{d / 2} \log \varepsilon \lesssim \varepsilon^{d / 2} \log \varepsilon
$$

as $\varepsilon \rightarrow 0$, since $\lim _{\varepsilon \rightarrow 0} \sigma_{\varepsilon}=\sigma>0$ in this case. This proves (1.6). Finally,

$$
\begin{aligned}
\left\langle h\left(\Phi_{\varepsilon}\right)-h(\sigma W)\right\rangle & =\left\langle h\left(\Phi_{\varepsilon}\right)-h\left(\sigma_{\varepsilon} W\right)\right\rangle+\left\langle h\left(\sigma_{\varepsilon} W\right)-h(\sigma W)\right\rangle \\
& =\sigma_{\varepsilon}\left\langle\hat{h}_{\varepsilon}\left(\sigma_{\varepsilon}^{-1} \Phi_{\varepsilon}\right)-\hat{h}_{\varepsilon}(W)\right\rangle+\left\langle h\left(\sigma_{\varepsilon} W\right)-h(\sigma W)\right\rangle \\
& =\sigma_{\varepsilon}\left\langle\hat{h}_{\varepsilon}\left(F_{\varepsilon}\right)-\hat{h}_{\varepsilon}(W)\right\rangle+\left\langle h\left(\sigma_{\varepsilon} W\right)-h(\sigma W)\right\rangle
\end{aligned}
$$

where $\hat{h}_{\varepsilon}(\cdot)=\sigma_{\varepsilon}^{-1} h\left(\sigma_{\varepsilon} \cdot\right)$ also satisfies $\left\|\hat{h}_{\varepsilon}^{\prime}\right\|_{\infty} \leq 1$. Hence

$$
\sup _{\left\|h^{\prime}\right\|_{\infty} \leq 1}\left\langle h\left(\Phi_{\varepsilon}\right)-h(\sigma W)\right\rangle \lesssim \varepsilon^{d / 2} \log \varepsilon+\left|\sigma_{\varepsilon}-\sigma\right|,
$$

which implies that (1.5) holds, as well. 
Proof of Lemma 2.2. If $|e|>2 \varepsilon^{-1}$ and $|x| \leq \varepsilon^{-1}$, then $|x-e|>|e| / 2 \geq 1$. So, clearly

$$
\sum_{|x| \leq \varepsilon^{-1}} \frac{1}{(1+|x-e|)^{d-1}} \lesssim \frac{\varepsilon^{-d}}{|e|^{d-1}}=\frac{\varepsilon^{-1}}{|\varepsilon e|^{d-1}}
$$

in this case. For $|e|<2 \varepsilon^{-1}$,

$$
\sum_{|x| \leq \varepsilon^{-1}} \frac{1}{(1+|x-e|)^{d-1}} \leq \int_{|y|<3 \varepsilon^{-1}} \frac{1}{(1+|y|)^{d-1}} d y \lesssim \varepsilon^{-1} .
$$

So, (2.13) holds in this case, as well.

Proof of Lemma 2.3. First, consider the sum over edges satisfying $|e|<\varepsilon^{-1}$ :

$$
\begin{aligned}
\sum_{|e|<\varepsilon^{-1}} \frac{1}{\left(1+\left|e-e^{\prime}\right|\right)^{d}} \frac{1}{(1+|\varepsilon e|)^{p}} & \lesssim \sum_{|e|<\varepsilon^{-1}} \frac{1}{\left(1+\left|e-e^{\prime}\right|\right)^{d}} \\
& \lesssim \begin{cases}|\log \varepsilon|, \quad \forall e^{\prime} \\
\frac{\varepsilon^{-d}}{\left|e^{\prime}\right|^{d}}, \quad \forall\left|e^{\prime}\right|>\varepsilon^{-1}\end{cases} \\
& \lesssim \frac{|\log \varepsilon|}{\left(1+\left|\varepsilon e^{\prime}\right|\right)^{d}} .
\end{aligned}
$$

Next, consider the sum over edges satisfying $|e|>\varepsilon^{-1}$ :

$$
\begin{aligned}
\sum_{|e| \geq \varepsilon^{-1}} \frac{1}{\left(1+\left|e-e^{\prime}\right|\right)^{d}} \frac{1}{(1+|\varepsilon e|)^{p}} & \lesssim \sum_{|e| \geq \varepsilon^{-1}} \frac{1}{\left(1+\left|e-e^{\prime}\right|\right)^{d}} \frac{1}{|\varepsilon e|^{p}} \\
& \lesssim \varepsilon^{-p} \int_{|x|>\varepsilon^{-1}} \frac{1}{\left(1+\left|x-e^{\prime}\right|\right)^{d}} \frac{1}{|x|^{p}} d x .
\end{aligned}
$$

Let $x=\varepsilon^{-1} z, e^{\prime}=\varepsilon^{-1} w$. Then

$$
\sum_{|e| \geq \varepsilon^{-1}} \frac{1}{\left(1+\left|e-e^{\prime}\right|\right)^{d}} \frac{1}{(1+|\varepsilon e|)^{p}} \lesssim \varepsilon^{-d} \int_{|z|>1} \frac{1}{\left(1+\varepsilon^{-d}|z-w|^{d}\right)} \frac{1}{|z|^{p}} d z
$$

Restricting the integral in (2.20) to the set $|z-w| \leq \varepsilon$, we have

$$
\varepsilon^{-d} \int_{\substack{|z|>1 \\|z-w| \leq \varepsilon}} \frac{1}{\left(1+\varepsilon^{-d}|z-w|^{d}\right)} \frac{1}{|z|^{p}} d z \lesssim \varepsilon^{-d} \int_{\substack{|z|>1 \\|z-w| \leq \varepsilon}} \frac{1}{|z|^{p}} d z \lesssim \min \left(1,|w|^{-p}\right) .
$$

Restricting the integral in (2.20) to the set $|z-w| \geq \varepsilon$, we have

$$
\varepsilon^{-d} \int_{|z|>1} \frac{1}{\left(1+\varepsilon^{-d}|z-w|^{d}\right)} \frac{1}{|z|^{p}} d z \lesssim \int_{|z-w|>1} \frac{1}{|z-w|^{d}} \frac{1}{|z|^{p}} d z .
$$

If $|z|>3|w|$, then $|w-z|>|z| / 2$, so

$$
\int_{\substack{|z-w|>1 \\|z|>3|w|}} \frac{1}{|z-w|^{d}} \frac{1}{|z|^{p}} d z \leq \int_{\substack{|z|>1 \\|z|>3|w|}} \frac{1}{|z|^{p+d}} d z \lesssim \min \left(1,|w|^{-p}\right) .
$$

On the other hand, restricting to $|z|<3|w|$,

$$
\begin{aligned}
\int_{\substack{|z-w| \geq \varepsilon \\
|z|<3|w|}} \frac{1}{|z-w|^{d}} \frac{1}{|z|^{p}} d z & =\int_{\substack{\varepsilon \leq|z-w| \leq 1 \\
|z|<3|w|}} \frac{1}{|z-w|^{d}} \frac{1}{|z|^{p}} d z+\int_{\substack{|z-w| \geq 1 \\
|z|<3|w|}} \frac{1}{|z-w|^{d}} \frac{1}{|z|^{p}} d z \\
& \lesssim|\log \varepsilon| \min \left(1,|w|^{-p}\right)+\log (1+|w|) \min \left(1,|w|^{-p}\right) .
\end{aligned}
$$


The bound (2.15) now follows by combining these estimates with $w=\varepsilon e^{\prime}$.

\section{Proof of Proposition 1.3}

By [10], it suffices to show the following result.

Proposition 3.1. For $f \in C_{c}^{0}\left(\mathbb{R}^{d} ; \mathbb{R}\right)$, let $f_{\lambda}(x)=\lambda^{-d} f(x / \lambda)$. For all $p \geq 1$, there exists a constant $C=C(p, f)$ such that for all $\varepsilon, \lambda \in(0,1]$,

$$
\left\langle\left|\Phi_{\varepsilon}\left(f_{\lambda}\right)\right|^{p}\right\rangle^{1 / p} \leq C \lambda^{1-\frac{d}{2}}
$$

Proof. Observe that for any $\varepsilon, \lambda \in(0,1]$,

$$
\Phi_{\varepsilon}\left(f_{\lambda}\right)=\lambda^{1-\frac{d}{2}} \Phi_{r}(f)
$$

with $r=\varepsilon / \lambda$. Therefore, to prove Proposition 3.1, it suffices to show that

$$
\left\langle\left|\Phi_{\varepsilon}(f)\right|^{p}\right\rangle^{1 / p} \leq C
$$

holds for all $\varepsilon>0$.

Since $\phi$ is stationary and $\langle\phi(0)\rangle=0$, we know that $\left\langle\Phi_{\varepsilon}(f)\right\rangle=0$. In particular,

$$
\left\langle\Phi_{\varepsilon}(f)^{p}\right\rangle^{2} \lesssim 1 \quad \text { uniformly over } \varepsilon>0
$$

holds for $p=1$. Arguing inductively, let us suppose that (3.2) holds for some positive integer $p=n$. We claim that (3.2) must also hold for $p=2 n$. To prove this claim, we use the identity

$$
\left\langle\Phi_{\varepsilon}(f)^{2 n}\right\rangle=\left\langle\Phi_{\varepsilon}(f)^{n}\right\rangle^{2}+\operatorname{Var}\left(\Phi_{\varepsilon}(f)^{n}\right),
$$

which, by the induction hypothesis, gives us

$$
\left\langle\Phi_{\varepsilon}(f)^{2 n}\right\rangle \lesssim 1+\operatorname{Var}\left(\Phi_{\varepsilon}(f)^{n}\right) .
$$

We will show that $\operatorname{Var}\left(\Phi_{\varepsilon}(f)^{n}\right) \lesssim\left\langle\Phi_{\varepsilon}(f)^{2 n}\right\rangle^{1-1 / n}$, hence

$$
\left\langle\Phi_{\varepsilon}(f)^{2 n}\right\rangle \lesssim 1+\left\langle\Phi_{\varepsilon}(f)^{2 n}\right\rangle^{1-1 / n} .
$$

This bound and Young's inequality establish the claim that (3.2) also holds for $p=2 n$.

To prove that $\operatorname{Var}\left(\Phi_{\varepsilon}(f)^{n}\right) \lesssim\left\langle\Phi_{\varepsilon}(f)^{2 n}\right\rangle^{1-1 / n}$, we apply the spectral gap inequality and Hölder's inequality:

$$
\begin{aligned}
\operatorname{Var}\left(\Phi_{\varepsilon}(f)^{n}\right) & \lesssim \sum_{e}\left\langle\left|\partial_{e} \Phi_{\varepsilon}(f)^{n}\right|^{2}\right\rangle \\
& \lesssim \sum_{e}\left\langle\left|\Phi_{\varepsilon}(f)^{n-1} \partial_{e} \Phi_{\varepsilon}(f)\right|^{2}\right\rangle \\
& \leq \sum_{e}\left\langle\Phi_{\varepsilon}(f)^{2 n}\right\rangle^{1-1 / n}\left\langle\left|\partial_{e} \Phi_{\varepsilon}(f)\right|^{2 n}\right\rangle^{1 / n} \\
& =\left\langle\Phi_{\varepsilon}(f)^{2 n}\right\rangle^{1-1 / n} \sum_{e}\left\langle\left|\partial_{e} \Phi_{\varepsilon}(f)\right|^{2 n}\right\rangle^{1 / n} .
\end{aligned}
$$

Now we show that the last sum is bounded by a constant. Without loss of generality, assume that $f(x)=0$ for $|x|>1$. Recall that $\left\langle\left|\partial_{e} \phi(x)\right|^{p}\right\rangle^{1 / p} \lesssim(1+|x-e|)^{1-d}$ (see (2.10)). So,

$$
\begin{aligned}
\left\langle\left(\partial_{e} \Phi(f)\right)^{2 n}\right\rangle^{1 /(2 n)} & =\varepsilon^{\frac{d}{2}+1}\left\langle\left(\sum_{x} f(\varepsilon x) \partial_{e} \phi(x)\right)^{2 n}\right\rangle^{1 /(2 n)} \\
& \lesssim \varepsilon^{\frac{d}{2}+1} \sum_{|x| \leq \varepsilon^{-1}}\left\langle\left(\partial_{e} \phi(x)\right)^{2 n}\right\rangle^{1 /(2 n)} \\
& \lesssim \varepsilon^{\frac{d}{2}+1} \sum_{|x| \leq \varepsilon^{-1}} \frac{1}{(1+|x-e|)^{d-1}}
\end{aligned}
$$


The last sum is controlled by Lemma 2.2, which leads to

Therefore,

$$
\left\langle\left(\partial_{e} \Phi(f)\right)^{2 n}\right\rangle^{1 /(2 n)} \leq \varepsilon^{\frac{d}{2}+1} \frac{\varepsilon^{-1}}{(1+|\varepsilon e|)^{d-1}} .
$$

$$
\sum_{e}\left\langle\left|\partial_{e} \Phi_{\varepsilon}(f)\right|^{2 n}\right\rangle^{1 / n} \lesssim \varepsilon^{d+2} \sum_{e} \frac{\varepsilon^{-2}}{(1+|\varepsilon e|)^{2 d-2}} \lesssim 1
$$

since $d \geq 3$. In view (3.5), we have now established that $\operatorname{Var}\left(\Phi_{\varepsilon}(f)^{n}\right) \lesssim\left\langle\Phi_{\varepsilon}(f)^{2 n}\right\rangle^{1-1 / n}$.

By induction on $n$, we have proved that

$$
\left\langle\Phi_{\varepsilon}(f)^{p}\right\rangle^{2} \lesssim 1
$$

holds for all $p \in\left\{2^{n} \mid n=0,1,2,3, \ldots\right\}$. Now (3.1) follows by Jensen's inequality.

\section{Stein's Method}

In this section, we prove Proposition 2.1, which is a version of Theorem 2.2 in [4] and Theorem 3.1 (and Remark 3.6) in [12], stated in a form that is convenient for our purpose. The basis of the estimate is the following lemma:

Lemma 4.1 (See [3], Lemma 4.2). Suppose $h: \mathbb{R} \rightarrow \mathbb{R}$ is absolutely continuous with bounded derivative, and $Y \sim N(0,1)$. There exists a solution to

$$
\phi^{\prime}(x)-x \phi(x)=h(x)-\langle h(Y)\rangle, \quad x \in \mathbb{R}
$$

which satisfies $\left\|\phi^{\prime}\right\|_{\infty} \leq \sqrt{\frac{2}{\pi}}\left\|h^{\prime}\right\|_{\infty}$ and $\left\|\phi^{\prime \prime}\right\|_{\infty} \leq 2\left\|h^{\prime}\right\|_{\infty}$.

Proof of Proposition 2.1. Let $F \in L^{2}(\Omega)$ be such that $\langle F\rangle=0,\left\langle F^{2}\right\rangle=1$. By (4.1), we have

$$
\begin{aligned}
\langle h(F)-h(Y)\rangle & =\left\langle\phi^{\prime}(F)-\phi(F) F\right\rangle \\
& =\operatorname{Cov}\left(\left\langle\phi^{\prime}(F)\right\rangle F-\phi(F), F\right) .
\end{aligned}
$$

Since $\partial_{e} F \in L^{2}(\Omega)$ for all $e \in \mathbb{B}$, we can estimate this covariance by applying the Helffer-Sjöstrand correlation representation (see [11], Prop. 3.1) to $\operatorname{Cov}(G, F)$ with $G=\left\langle\phi^{\prime}(F)\right\rangle F-\phi(F)$. Let $\partial_{e}^{*}=-\partial_{e}+\zeta_{e}$ be the adjoint of the derivative operator $\partial_{e}$. Let $\mathscr{L}=\partial^{*} \partial$, where $\partial F=\left(\partial_{e} F\right)_{e \in \mathbb{B}}$ and for $K=\left(K_{e}\right)_{e \in \mathbb{B}}, \partial^{*} K=\sum_{e} \partial_{e}^{*} K_{e}$. From the correlation representation, we obtain

$$
\begin{aligned}
\langle h(F)-h(Y)\rangle & =\sum_{e}\left\langle\partial_{e} G(\mathscr{L}+1)^{-1} \partial_{e} F\right\rangle \\
& =\sum_{e}\left\langle\left(\left\langle\phi^{\prime}(F)\right\rangle-\phi^{\prime}(F)\right) \partial_{e} F(\mathscr{L}+1)^{-1} \partial_{e} F\right\rangle .
\end{aligned}
$$

Observe that $\left\langle\phi^{\prime}(F)\right\rangle-\phi^{\prime}(F) \in L^{\infty}$ has zero mean. Moreover, the series $\sum_{e} \partial_{e} F(\mathscr{L}+1)^{-1} \partial_{e} F$ converges in $L^{1}(\Omega)$ and has mean $1=\operatorname{Cov}(F, F)$. Hence

$$
\begin{aligned}
\langle h(F)-h(Y)\rangle & =\left\langle\left(\left\langle\phi^{\prime}(F)\right\rangle-\phi^{\prime}(F)\right)\left(\sum_{e} \partial_{e} F(\mathscr{L}+1)^{-1} \partial_{e} F\right)\right\rangle \\
& =\left\langle\phi^{\prime}(F)\left(1-\sum_{e} \partial_{e} F(\mathscr{L}+1)^{-1} \partial_{e} F\right)\right\rangle \\
& \leq\left\|\phi^{\prime}\right\|_{\infty}\left\langle\left|1-\sum_{e} \partial_{e} F(\mathscr{L}+1)^{-1} \partial_{e} F\right|\right\rangle \\
& \leq \sqrt{\frac{2}{\pi}}\left\|h^{\prime}\right\|_{\infty}\left\langle\left|1-\sum_{e} \partial_{e} F(\mathscr{L}+1)^{-1} \partial_{e} F\right|\right\rangle
\end{aligned}
$$


Assuming that $\sum_{e}\left\langle\left|\partial_{e} F\right|^{4}\right\rangle^{1 / 2}<\infty$, we claim that the sum

$$
S=\sum_{e} \partial_{e} F(\mathscr{L}+1)^{-1} \partial_{e} F
$$

is in $L^{2}(\Omega)$, and we will estimate the last term in (4.3) by the variance

$$
\left\langle\left|1-\sum_{e} \partial_{e} F(\mathscr{L}+1)^{-1} \partial_{e} F\right|\right\rangle \leq \sqrt{\operatorname{Var}\left(\sum_{e} \partial_{e} F(\mathscr{L}+1)^{-1} \partial_{e} F\right)} .
$$

Indeed,

$$
\left\|\partial_{e} F(\mathscr{L}+1)^{-1} \partial_{e} F\right\|_{2} \leq\left\|\partial_{e} F\right\|_{4}\left\|(\mathscr{L}+1)^{-1} \partial_{e} F\right\|_{4} \leq\left\|\partial_{e} F\right\|_{4}^{2},
$$

since $(\mathscr{L}+1)^{-1}$ is a contraction on $L^{p}(\Omega)$ for any $p \geq 2$ (see Prop. 3.2 of [11]). Thus, $S \in L^{2}(\Omega)$ if $\sum_{e}\left\|\partial_{e} F\right\|_{4}^{2}<\infty$.

Under the additional assumption $\left\langle\left|\partial_{e^{\prime}} \partial_{e} F\right|^{4}\right\rangle<\infty$ for all $e, e^{\prime} \in \mathbb{B}$, we also have $\partial_{e^{\prime}} S \in L^{2}(\Omega)$ for all $e^{\prime} \in \mathbb{B}$, so that $\operatorname{Var}(S)$ can be estimated by the Gaussian spectral gap inequality. We compute:

$$
\partial_{e^{\prime}}(\mathscr{L}+1)^{-1} \partial_{e} F=(\mathscr{L}+2)^{-1} \partial_{e^{\prime}} \partial_{e} F,
$$

so that

$$
\partial_{e^{\prime}}\left(\partial_{e} F(\mathscr{L}+1)^{-1} \partial_{e} F\right)=\left(\partial_{e^{\prime}} \partial_{e} F\right)(\mathscr{L}+1)^{-1} \partial_{e} F+\partial_{e} F(\mathscr{L}+2)^{-1} \partial_{e^{\prime}} \partial_{e} F .
$$

The operator $(\mathscr{L}+2)^{-1}$ is also a contraction, satisfying $\left\|(\mathscr{L}+2)^{-1} u\right\|_{p} \leq(1 / 2)\|u\|_{p}$ for all $u \in L^{p}(\Omega), p \geq 2$ (this follows from minor modification to the proof of Prop. 3.2 of [11]). Therefore,

$$
\begin{aligned}
\left\|\partial_{e^{\prime}}\left(\partial_{e} F(\mathscr{L}+1)^{-1} \partial_{e} F\right)\right\|_{2} & \leq\left\|\left(\partial_{e^{\prime}} \partial_{e} F\right)(\mathscr{L}+1)^{-1} \partial_{e} F\right\|_{2}+\left\|\partial_{e} F(\mathscr{L}+2)^{-1} \partial_{e^{\prime}} \partial_{e} F\right\|_{2} \\
& \leq\left\|\partial_{e^{\prime}} \partial_{e} F\right\|_{4}\left\|(\mathscr{L}+1)^{-1} \partial_{e} F\right\|_{4}+\left\|\partial_{e} F\right\|_{4}\left\|(\mathscr{L}+2)^{-1} \partial_{e^{\prime}} \partial_{e} F\right\|_{4} \\
& \leq\left(1+\frac{1}{2}\right)\left\|\partial_{e^{\prime}} \partial_{e} F\right\|_{4}\left\|\partial_{e} F\right\|_{4}<\infty .
\end{aligned}
$$

Hence, $\partial_{e^{\prime}} S \in L^{2}(\Omega)$ for all $e^{\prime} \in \mathbb{B}$.

Now we apply the Gaussian spectral gap inequality:

$$
\begin{aligned}
\operatorname{Var}\left(\sum_{e} \partial_{e} F(\mathscr{L}+1)^{-1} \partial_{e} F\right) \leq & \sum_{e^{\prime}}\left\langle\left|\partial_{e^{\prime}} \sum_{e} \partial_{e} F(\mathscr{L}+1)^{-1} \partial_{e} F\right|^{2}\right\rangle \\
\leq & 2 \sum_{e^{\prime}}\left\langle\left|\sum_{e}\left(\partial_{e^{\prime}} \partial_{e} F\right)(\mathscr{L}+1)^{-1} \partial_{e} F\right|^{2}\right\rangle \\
& +2 \sum_{e^{\prime}}\left\langle\left|\sum_{e} \partial_{e} F(\mathscr{L}+2)^{-1} \partial_{e^{\prime}} \partial_{e} F\right|^{2}\right\rangle .
\end{aligned}
$$

To bound the last two sums, we apply Minkowski's inequality:

$$
\begin{gathered}
\left\langle\left|\sum_{e}\left(\partial_{e^{\prime}} \partial_{e} F\right)(\mathscr{L}+1)^{-1} \partial_{e} F\right|^{2}\right\rangle^{1 / 2} \leq \sum_{e}\left\langle\left|\left(\partial_{e^{\prime}} \partial_{e} F\right)(\mathscr{L}+1)^{-1} \partial_{e} F\right|^{2}\right\rangle^{1 / 2}, \\
\left\langle\left|\sum_{e}\left(\partial_{e^{\prime}} \partial_{e} F\right)(\mathscr{L}+1)^{-1} \partial_{e} F\right|^{2}\right\rangle^{1 / 2} \leq \sum_{e}\left\langle\left|\partial_{e} F(\mathscr{L}+2)^{-1} \partial_{e^{\prime}} \partial_{e} F\right|^{2}\right\rangle^{1 / 2} .
\end{gathered}
$$

As was seen in (4.5), we have

$$
\begin{aligned}
\left\langle\left|\left(\partial_{e^{\prime}} \partial_{e} F\right)(\mathscr{L}+1)^{-1} \partial_{e} F\right|^{2}\right\rangle^{1 / 2} & \leq\left\langle\left|\partial_{e^{\prime}} \partial_{e} F\right|^{4}\right\rangle^{1 / 4}\left\langle\left|\partial_{e} F\right|^{4}\right\rangle^{1 / 4} \\
\left\langle\left|\partial_{e} F(\mathscr{L}+2)^{-1} \partial_{e^{\prime}} \partial_{e} F\right|^{2}\right\rangle^{1 / 2} & \leq \frac{1}{2}\left\langle\left|\partial_{e} F\right|^{4}\right\rangle^{1 / 4}\left\langle\left|\partial_{e^{\prime}} \partial_{e} F\right|^{4}\right\rangle^{1 / 4}
\end{aligned}
$$


Therefore, returning to (4.6), we obtain that

$$
\operatorname{Var}\left(\sum_{e} \partial_{e} F(\mathscr{L}+1)^{-1} \partial_{e} F\right) \leq \frac{5}{2} \sum_{e^{\prime}}\left(\sum_{e}\left\langle\left|\partial_{e} F\right|^{4}\right\rangle^{1 / 4}\left\langle\left|\partial_{e^{\prime}} \partial_{e} F\right|^{4}\right\rangle^{1 / 4}\right)^{2},
$$

and thus from (4.3) and (4.4) we conclude

$$
\langle h(F)-h(Y)\rangle \leq \sqrt{\frac{5}{\pi}}\left\|h^{\prime}\right\|_{\infty} \sqrt{\sum_{e^{\prime}}\left(\sum_{e}\left\langle\left|\partial_{e} F\right|^{4}\right\rangle^{1 / 4}\left\langle\left|\partial_{e^{\prime}} \partial_{e} F\right|^{4}\right\rangle^{1 / 4}\right)^{2}} .
$$

(The assumptions on $F$ in Proposition 2.1 do not guarantee that the right side of (4.8) is finite. In the case that the right side is infinite, the conclusion of the theorem holds trivially.)

\section{ACKNOWLEDGEMENTS}

JN acknowledges financial support from US National Science Foundation grants DMS-1007572 and DMS-1351653.

\section{REFERENCES}

[1] N. Berger and M. Biskup, Quenched invariance principle for simple random walk on percolation clusters. Probab. Theory Related Fields 137 (2007), pp. 83-120.

[2] M. Biskup, Recent progress on the random conductance model, Prob. Surveys 8 (2011), pp. 294-373.

[3] S. Chatterjee, A new method of normal approximation, Ann. Probab. 36 (2008), pp. 1584-1610.

[4] S. Chatterjee, Fluctuations of eigenvalues and second order Poincaré inequalities, Prob. Theory Related Fields 143 (2009), pp. 1-40.

[5] A. Gloria and F. Otto. An optimal variance estimate in stochastic homogenization of discrete elliptic equations. Ann. Probab. 39 (2011), pp. 779-856.

[6] A. Gloria, S. Neukamm, and F. Otto. Quantifcation of ergodicity in stochastic homogenization: optimal bounds via spectral gap on Glauber dynamics. Invent. Math. 199 (2015), pp. 455-515.

[7] S.M. Kozlov, The averaging of random operators, Math. USSR Sb. 109 (1979), pp. 188-202.

[8] R. Künneman, The diffusion limit for reversible Jump processes on $\mathbb{Z}^{d}$ with ergodic random bond conductivities, Comm. Math. Phys. 90 (1983), pp. 27-68.

[9] D. Marahrens and F. Otto. Annealed estimates on the Green function, Probab. Theory Relat. Fields, to appear. http://dx.doi.org/10.1007/s00440-014-0598-0

[10] J.-C. Mourrat. A tightness criterion in local Hölder spaces of negative regularity. Preprint (2015). http: //arxiv.org/abs/1502.07315

[11] J.-C. Mourrat and F. Otto. Correlation structure of the corrector in stochastic homogenization. Preprint (2014). http://arxiv.org/abs/1402.1924

[12] I. Nourdin and G. Peccati, Stein's method on Wiener chaos, Probab. Theory Relat. Fields 145 (2009), p. 75-118.

[13] G.C. Papanicolaou and S.R.S. Varadhan, Boundary value problems with rapidly oscillating random coefficients. in Random Fields Vol. I, II (Esztergom, 1979), Colloq. Math. Soc. János Bolyai, 27 North Holland, New York: 1981. pp. 835- 873 .

[14] J.M. Steele, An Efron-Stein inequality for nonsymmetric statistics, Ann. Statist. 14 (1986), pp. $753-758$.

[15] C. Stein, Approximate Computation of Expectations. IMS Lecture Notes - Monograph Series 7. IMS, Hayward, CA., 1972.

(Jean-Christophe Mourrat) Ecole normale supérieure de Lyon, CNRS, Lyon, France

E-mail address: jean-christophe.mourrat@ens-lyon.fr

(James Nolen) Department of Mathematics, Duke University, Durham, North Carolina, USA

E-mail address: nolen@math.duke.edu 